\title{
Naturally Occurring Mutations to Muscle-Type Creatine Kinase Impact Its Canonical and Pharmacological Activities in a Substrate-Dependent Manner In Vitro
}

\author{
Eric P. Mosher, Colten D. Eberhard, and Namandjé N. Bumpus
}

Department of Pharmacology and Molecular Sciences, Johns Hopkins University School of Medicine, Baltimore, Maryland

Received June 22, 2021; accepted September 7, 2021

\begin{abstract}
Tenofovir (TFV) is a key component of human immunodeficiency virus (HIV) pre-exposure prophylaxis (PrEP). TFV is a nucleotide analog reverse-transcriptase inhibitor prodrug that requires two separate phosphorylation reactions by intracellular kinases to form the active metabolite tenofovir-diphosphate (TFV-DP). Muscle-type creatine kinase (CKM) has previously been demonstrated to be the kinase most responsible for the phosphorylation of tenofovir-monophosphate (TFV-MP) to the active metabolite in colon tissue. Because of the importance of CKM in TFV activation, genetic variation in CKM may contribute to interindividual variability in TFV-DP levels. In the present study, we report 10 naturally occurring CKM mutations that reduced TFV-MP phosphorylation in vitro: T35I, R43Q, I92M, H97Y, R130H, R132C, F169L, Y173C, W211R, V280L, and N286l. Interestingly, of these 10 , only $4-\mathrm{R} 130 \mathrm{H}$, R132C, W211R, and N286I-reduced both canonical CKM activities: ADP phosphorylation and ATP dephosphorylation. Although positions 130, 132, and 286 are located in the active site, the other mutations that resulted in decreased TFV-MP phosphorylation occur elsewhere in the protein structure. Four of these eight
\end{abstract}

\section{Introduction}

The global rate of new HIV infections per year has been declining for the last 20 years, and mortality has decreased over the last 15 years (GBD 2017 HIV collaborators, 2019). Still, it is estimated that 1.7 million people became newly infected and 690,000 AIDS-related deaths occurred in 2019 (Joint United Nations Program on HIV/AIDS, 2020). A more recent advancement, HIV pre-exposure prophylaxis (PrEP), has been demonstrated to reduce someone's risk of acquiring HIV (Baeten et al.,

This work was supported by National Institutes of Health (NIH) National Institute of Allergy and Infectious Diseases [Grant R01AI128781] and National Institute on Aging [Grant R01AG064908] (to N.N.B.) and a 2020 PhRMA Foundation Predoctoral Fellowship in Pharmacology and Toxicology awarded to E.P.M. E.P.M. was also supported by NIH National Institute of General Medical Sciences [Grant T32-GM008763].

The authors report no conflict of interest.

dx.doi.org/10.1124/molpharm.121.000348.

S This article has supplemental material available at molpharm. aspetjournals.org.
mutations-T35I, R43Q, I92M, and W211R-were found to decrease the thermal stability of the protein. Additionally, the W211R mutation was found to impact protein structure both locally and at a distance. These data suggest a substrate-specific effect such that certain mutations are tolerated for canonical activities while being deleterious toward the pharmacological activity of TFV activation, which could influence PrEP outcomes.

\section{SIGNIFICANCE STATEMENT}

Muscle-type creatine kinase (CKM) is important to the activation of tenofovir, a key component of HIV prophylaxis. This study demonstrates that naturally occurring CKM mutations impact enzyme function in a substrate-dependent manner such that some mutations that do not reduce canonical activities lead to reductions in the pharmacologically relevant activity. This finding at the intersection of drug metabolism and energy metabolism is important to the perspective on pharmacology of other drugs acted on by atypical drug-metabolizing enzymes.

ABBREVIATIONS: Arb, arbitrary units; BME, $\beta$-mercaptoethanol; CKM, muscle-type creatine kinase; dd-MS2, data-dependent; DMHA, $N, N$ dimethylhexylamine; DTT, dithioerythritol; HDX-MS, hydrogen-deuterium exchange mass spectrometry; HIV, human immunodeficiency virus; MS/MS, tandem mass spectrometry; $\mathrm{MS}_{\mathrm{MST}}$, melting temperature; PCR, polymerase chain reaction; PDB, Protein Data Bank; PrEP, preexposure prophylaxis; TFV, tenofovir; TFV-DP, TFV-diphosphate; TFV-MP, TFV-monophosphate; uHPLC-MS/MS, ultra-high performance liquid chromatography MS/MS; WT, wild type. 
However, even high-adherence subgroups do not achieve $100 \%$ protection (Grant et al., 2010; Baeten et al., 2012).

TFV is a prodrug that must be phosphorylated twice by intracellular kinases to become the active TFV-diphosphate (TFV-DP) metabolite. The effectiveness of PrEP relies on acting as a barrier, as intracellular TFV-DP levels in colon tissue, a potential site of exposure, correlate with HIV protection (Richardson-Harman et al., 2014). In addition to variability of plasma TFV, variability in mucosal tissue TFVDP has also been observed (Anderson et al., 2012; Louissaint et al., 2013). TFV activation is carried out by different kinases in different tissues (Lade et al., 2015). In colon tissue, adenylate kinase 2 performs the first phosphorylation reaction, converting TFV to TFV-monophosphate (TFV-MP). The second phosphorylation step is primarily performed by muscle-type creatine kinase (CKM). It is possible that person-toperson differences in the activity of these kinases are responsible for the observed TFV-DP variability. Multiple studies have reported on genetic variation in these three kinases in people receiving PrEP (Lade et al., 2015; Figueroa et al., $2018 \mathrm{a}, \mathrm{b})$. The discovery of naturally occurring mutations to the kinases important to TFV activation may lead to a more personalized approach to PrEP.

Creatine kinase enzymes are important to cellular energy metabolism, as they buffer intracellular ATP concentrations by storing and using high-energy phosphate species in the form of phosphocreatine (Wallimann et al., 1992, 2011). In addition to the Lade primary tissue study, Varga and Koch also demonstrated that CKM can catalyze TFV-MP phosphorylation in vitro (Koch et al., 2009; Varga et al., 2013; Lade et al., 2015). Three previous studies of the genetic variation of TFV-activating kinases in HIV prevention clinical trial participants found 31 mutations predicted by in silico tools to be damaging to CKM function (Lade et al., 2015; Figueroa et al., 2018a,b). It is possible that variability in CKM function due to genetics may influence TFV-DP formation in colon tissue, which may impact the efficacy of TFVbased PrEP.

In this work, we determine the impact of naturally occurring mutations on the ability of CKM to phosphorylate TFVMP to TFV-DP in vitro. The effect of these mutations on the canonical functions of the enzyme and protein stability and structure were further studied. It was found that several naturally occurring CKM mutations decreased enzyme function, although a substrate-specific effect was observed. Some mutations that decreased TFV-MP phosphorylation did not decrease activity toward endogenous substrates. Interestingly, four mutations reduced all activities. Three of these mutations are to active-site positions implicated in ligand binding. The other mutation, W211R, is outside of the active site and likely impacts the stability and structure of the protein. We have demonstrated that naturally occurring mutations to CKM lead to decreased TFV-MP phosphorylation in vitro. Importantly, the substrate-dependent effect of these mutations reveals that reduced canonical activity is not sufficient to predict decreased pharmacologically relevant activity for an atypical drug-metabolizing enzyme, such as CKM.

\section{Materials and Methods}

Materials/Chemicals. TFV-MP and TFV-DP were purchased from Toronto Research Chemicals, Inc. (North York, ON, Canada).
Solvents used were high-performance liquid chromatography-grade and purchased from Fisher Scientific (Hampton, NH). Other reagents used were reagent-grade and purchased from SigmaAldrich (St. Louis, MO) unless otherwise specified.

Cloning and Site-Directed Mutagenesis. Human muscle-type creatine kinase cDNA (OriGene, Rockville, MD) was inserted into a pET-30a vector (Sigma-Aldrich, St. Louis, MO) by polymerase incomplete primer extension cloning such that the expressed product possesses an N-terminal His-tag (Klock et al., 2008). Site-directed mutagenesis was performed using the QuikChange Lightning sitedirected mutagenesis kit (Agilent, Santa Clara, CA) following the manufacturer's instructions. The sequences of the primers used, which were purchased from Integrated DNA Technologies (Coralville, IA), are provided in Supplemental Table 1. Fifteen mutations found in the sequencing data from the aforementioned sequencing studies were selected, with a priority for the mutations predicted to likely be damaging to enzyme function by SIFT and PolyPhen prediction tools (Ng and Henikoff, 2001; Adzhubei et al., 2010; Lade et al., 2015; Figueroa et al., 2018a,b). Those predicted to decrease enzyme activity were T35I, T52N, I92M, H97Y, R130H, R132C, Y173C, W211R, F250S, N274Y, N286I, and L317M. Additionally, three mutations predicted to be benign were also included: R43Q, F169L, and V280L. Sequences of constructs were confirmed by Sanger DNA sequencing.

Recombinant Protein Expression and Purification. Plasmids were transformed into BL21 (DE3) pLysS competent Escherichia coli (Agilent, Santa Clara, CA). Cultures were grown in 11 Luria Broth containing $50 \mu \mathrm{g} / \mathrm{ml}$ kanamycin at $37^{\circ} \mathrm{C}$ with shaking for 2 hours, cooled to $4^{\circ} \mathrm{C}$ without shaking for 1 hour, and then induced with $200 \mu \mathrm{M}$ isopropyl $\beta$-D-1-thiogalactopyranoside and grown for 16 hours at $4^{\circ} \mathrm{C}$ with shaking. After growth, cells were pelleted by centrifugation at $5000 \times \mathrm{g}$.

Bacterial pellets were resuspended in a $50 \mathrm{mM}$ HEPES buffer at a $\mathrm{pH}$ of 7.3 containing $150 \mathrm{mM} \mathrm{NaCl}, 5 \%$ glycerol (v/v), $2 \mathrm{mM} \beta$-mercaptoethanol (BME), and 1X-EDTA-free protease inhibitor cocktail (Sigma-Aldrich, St. Louis, MO). Cells were then lysed by three passes through a microfluidizer (LM-10, Microfluidics, Westwood, MA). Cell debris was pelleted by centrifugation at $20,000 \times \mathrm{g}$ at $4^{\circ} \mathrm{C}$. Lysate supernatant was subsequently mixed with cobalt resin (Takara, Mountain View, CA) for 1 hour at $4^{\circ} \mathrm{C}$. The lysate/resin mixture was then loaded onto gravity flow columns (BioRad, Hercules, $\mathrm{CA}$ ), and unbound lysate contents were removed. The resin was then washed with 20 column volumes of low- and high-salt wash buffers (50 mM HEPES, pH 7.3, $150 \mathrm{mM} \mathrm{NaCl}, 5 \mathrm{mM}$ imidazole, $5 \%$ glycerol, and $2 \mathrm{mM}$ BME and $50 \mathrm{mM}$ HEPES, pH 7.3, $1 \mathrm{M} \mathrm{NaCl}, 5 \mathrm{mM}$ imidazole, $5 \%$ glycerol, $2 \mathrm{mM} \mathrm{BME}$, respectively). His-tagged CKM was eluted via stepwise addition of four column volumes of elution buffer (50 mM HEPES, pH 7.3, $150 \mathrm{mM} \mathrm{NaCl}, 5 \%$ glycerol, $2 \mathrm{mM}$ BME) containing increasing concentrations of imidazole $(10 \mathrm{mM}$ to $500 \mathrm{mM})$. After purification, CKM proteins were buffer-exchanged into storage buffer (20 mM HEPES, $\mathrm{pH} 7.3,150 \mathrm{mM} \mathrm{KCl}, 5 \%$ glycerol, $2 \mathrm{mM} \mathrm{BME}$ ) and concentrated using a $30-\mathrm{kDa}$ MWCO centrifugal filter (Sigma-Aldrich, St. Louis, MO) and frozen and stored at $-80^{\circ} \mathrm{C}$. Total protein yield ranged from $4-16 \mathrm{mg}$ per liter of culture. Protein concentration was determined by absorbance at a wavelength of $280 \mathrm{~nm}$ using a Take3 microvolume plate in a plate reader (BioTek Synergy HT, BioTek Instruments, Inc., Winooski, VT) and an extinction coefficient of $36747 \mathrm{M}^{-1} \mathrm{~cm}^{-1}$ determined using the ProtParam tool (Gasteiger et al., 2003). SDS-PAGE was performed to verify purity of the products (85\%-95\% purity by gel densitometry). The identity of purified wild-type (WT) CKM was validated by Western blot using a CKM-specific antibody (Proteintech, Rosemont, IL).

TFV-MP Phosphorylation Assay. Reactions were performed at a final volume of $200 \mu \mathrm{l}$ under the following buffer conditions: $75 \mathrm{mM}$ HEPES, $\mathrm{pH} 7.5,50 \mathrm{mM} \mathrm{KCl}, 5 \mathrm{mM} \mathrm{MgCl}_{2}, 2 \mathrm{mM}$ dithioerythritol (DTT), $500 \mathrm{nM}$ protein, and $100 \mu \mathrm{M}$ TFV-MP. The $100 \mu \mathrm{M}$ TFV-MP concentration was chosen to perform the assay under saturating 
conditions and minimize the reverse reaction. Solutions were prewarmed at $37^{\circ} \mathrm{C}$ for 5 minutes prior to initiation by the addition of phosphocreatine to a final concentration of $500 \mu \mathrm{M}$. After 30 minutes of incubation at $37^{\circ} \mathrm{C}$, reactions were quenched via direct addition of $1 \mathrm{ml}$ of ice-cold methanol followed by brief vortex mixing and incubation on ice for 10 minutes. Reactions were centrifuged at 10,000 rpm and $4{ }^{\circ} \mathrm{C}$ for 10 minutes. The resulting supernatants were removed and dried by vacuum centrifugation (Eppendorf, Hamburg, Germany). Control reactions were performed in parallel by omitting the addition of phosphocreatine. All reactions and controls were performed in triplicate.

Resulting TFV-DP formation was measured by ultra-high performance liquid chromatography tandem mass spectrometry (uHPLCMS/MS). Dried samples were reconstituted in $30 \mu \mathrm{l} 5 \mathrm{mM} N, N$-dimethylhexylamine (DMHA) in water, $\mathrm{pH}$ 7.0. Samples were subsequently analyzed on a Dionex Ultimate 3000 uHPLC (Thermo Fisher Scientific, Bremen, Germany) coupled to a TSQ Vantage triple quadrupole mass spectrometer (Thermo Fisher Scientific, Bremen, Germany). Mobile phase A was $5 \mathrm{mM}$ DMHA in water at $\mathrm{pH} 7.0$, and mobile phase B was $5 \mathrm{mM}$ DMHA in $50 \%$ acetonitrile $/ 50 \%$ water $(\mathrm{v} / \mathrm{v})$. Analytes were separated on a Halo C18 column $(2.1 \mathrm{~mm} \times 100 \mathrm{~mm}, 2.1 \mu \mathrm{m}$, Mac-Mod Analytical - Chadds Ford, PA) at a flow rate of $0.45 \mathrm{ml} / \mathrm{min}$ using the following gradient: $5 \% \mathrm{~B}$ from 0 to 1 minutes, $5 \%-45 \% \mathrm{~B}$ from 1 to 9 minutes, $45 \%$ B from 9 to 13 minutes, $45 \%-100 \%$ B from 13 to 15 minutes, $100 \%$ B from 15 to 20 minutes, $100 \%-5 \%$ B from 20 to 23 minutes, and 5\% B from 23 to 30 minutes. Analytes were ionized by heated electrospray ionization with a spray voltage of $5000 \mathrm{~V}$, capillary temperature of $381^{\circ} \mathrm{C}$, sheath gas flow rate of $50 \mathrm{Arb}$, and auxiliary gas flow rate of 55 Arb. TFV-DP was detected in positive ion mode using a single-reaction monitoring scan with a transition of mass to charge ratios of $448 \rightarrow 270$ with a collision energy of $35 \mathrm{~V}$. Chromatographic peak heights were used for relative comparisons of metabolite abundance. Statistics were performed comparing each mutant to wild-type CKM using a one-way ANOVA followed by a Dunnett multiple comparison test using GraphPad Prism.

ADP Phosphorylation Assay. Reactions were performed at a final volume of $200 \mu \mathrm{l}$ under the following buffer conditions: $75 \mathrm{mM}$ HEPES, $\mathrm{pH} 7.5,50 \mathrm{mM} \mathrm{KCl}, 5 \mathrm{mM} \mathrm{MgCl}_{2}, 2 \mathrm{mM}$ DTT, $500 \mathrm{nM}$ protein, and $100 \mu \mathrm{M}$ ADP. Solutions were prewarmed at $37^{\circ} \mathrm{C}$ for 5 minutes prior to initiation by the addition of phosphocreatine to a final concentration of $500 \mu \mathrm{M}$. After 30 minutes of incubation at $37^{\circ} \mathrm{C}$, reactions were quenched via direct addition of $1 \mathrm{ml}$ of ice-cold methanol followed by brief vortex mixing and incubation on ice for 10 minutes. Reactions were centrifuged at $10,000 \mathrm{rpm}$ and $4^{\circ} \mathrm{C}$ for 10 minutes. The resulting supernatants were removed and dried by vacuum centrifugation. Control reactions were performed in parallel by omitting the addition of phosphocreatine. All reactions and controls were performed in triplicate.

Detection of ATP formation was performed by uHPLC-MS/MS using the same column, mobile phases, and flow rate as the aforementioned TFV-DP detection method but a different gradient: $10 \% \mathrm{~B}$ from 0 to 1 minutes, $10 \%-45 \%$ B from 1 to 10 minutes, $45 \%$ B from 10 to 13 minutes, $45 \%-100 \%$ B from 13 to 14 minutes, $100 \%$ B from 14 to 18 minutes, $100 \%-10 \% \mathrm{~B}$ from 18 to 20 minutes, and $10 \% \mathrm{~B}$ from 20 to 25 minutes. Analytes were ionized by heated electrospray ionization with a spray voltage of $4500 \mathrm{~V}$, capillary temperature of $250^{\circ} \mathrm{C}$, sheath gas flow rate of $50 \mathrm{Arb}$, and auxiliary gas flow rate of 50 Arb. ATP was detected in negative ion mode using a single-reaction monitoring scan with a transition of mass to charge ratios of $506 \rightarrow 159$ with a collision energy of $34 \mathrm{~V}$. Chromatographic peak heights were used for relative comparisons of metabolite abundance. Statistics were performed comparing each mutant to wild-type CKM using a one-way ANOVA followed by a Dunnett multiple-comparisons test using GraphPad Prism.

Pyruvate Kinase/Lactate Dehydrogenase-Coupled ATP Dephosphorylation Kinetics. The impact of naturally occurring CKM mutations on ATP dephosphorylation activity was measured using an enzyme-coupled kinetic assay in which the ADP product of the CKM reaction was further acted on by a pyruvate kinase/lactate dehydrogenase system such that ADP production led to an equivalent conversion of $\mathrm{NADH}$ to $\mathrm{NAD}^{+}$and a subsequent loss of absorbance intensity at a wavelength of $340 \mathrm{~nm}$. Final assay conditions were as follows: $75 \mathrm{mM}$ HEPES, $\mathrm{pH} 7.5,55 \mathrm{mM} \mathrm{MgCl}_{2}, 50 \mathrm{mM} \mathrm{KCl}$, $2 \mathrm{mM}$ DTT, $400 \mu \mathrm{M}$ NADH, $1 \mathrm{mM}$ phosphoenolpyruvate, $20 \mathrm{mM}$ creatine, 66 units/ml pyruvate kinase, 105 units/ml lactate dehydrogenase, and $20 \mathrm{nM}$ CKM mutant. Ten different ATP concentrations were used: $50 \mathrm{mM}, 25 \mathrm{mM}, 12.5 \mathrm{mM}, 6.25 \mathrm{mM}, 3.13 \mathrm{mM}, 1.56 \mathrm{mM}$, $0.78 \mathrm{mM}, 0.39 \mathrm{mM}, 0.01 \mathrm{mM}$, and $0 \mathrm{mM}$. Assays were performed in 96-well plates at a volume of $100 \mu \mathrm{l}$ per well, with each well for a given mutant containing a different starting concentration of ATP. Measurements were made using a plate reader (BioTek Synergy HT, BioTek Instruments, Inc., Winooski, VT) kept at $37^{\circ} \mathrm{C}$ with live-time serial measurements and shaking in between reads. Absorbance at a wavelength of $340 \mathrm{~nm}$ was recorded every 10 seconds for the first 2 minutes and then every 30 seconds for the next 13.5 minutes. All assays were performed in triplicate. Linear fits were performed on the absorbance values in the first 2 minutes to determine initial rates (Microsoft Excel). Conversion between absorbance and concentration units was performed based on an NADH standard curve. Michaelis-Menten plots were generated using GraphPad Prism. Data were globally fit using all points from each replicate with an unweighted least-squares regression to the traditional MichaelisMenten equation with two variables $\left(V_{\max }\right.$ and $\left.K_{m}\right)$. Error values for the $\mathrm{k}_{\mathrm{cat}} / \mathrm{K}_{\mathrm{m}}$ ratio were determined by propagation of the standard error values from the individual components provided by the nonlinear fit as previously described (Johnson, 2019). Because of the lack of a $v_{0}$ plateau at the ATP concentrations used and the resulting poor rectangular hyperbola fit for the R132C mutant data, a linear fit was used instead to calculate $\mathrm{k}_{\mathrm{cat}} / \mathrm{K}_{\mathrm{m}}$ for this mutant (GraphPad Prism).

Molecular Docking Simulations. A homology model structure of wild-type human CKM was generated using the human CKM peptide sequence modeled to the structure of rabbit CKM structure cocrystalized with ligands that mimic the transition state of phosphoryl transfer between creatine and ADP (PDB 1U6R) (Ohren et al., 2007). First, the side-chain positions in the rabbit structure were energyminimized using the YASARA energy minimization server (Krieger et al., 2009). Then, the homology model was generated using the SWISS-MODEL web server (Waterhouse et al., 2018). The resulting structure was used as the receptor for docking simulations. The receptor was further prepared for docking using AutoDock 4.2 to add polar hydrogens and Gasteiger charges (Morris et al., 2009). For preparation of the ADP and TFV-MP ligands, structure data files were obtained from the PubChem database (compound identification numbers 6022 and 6320284 for ADP and TFV-MP, respectively). Ligands were converted to the PDBQT file format using OpenBabel (O’Boyle et al., 2011). Ligands were separately docked to the rigid receptor using AutoDock 4.2 using a $120 \times 120 \times 120$-point grid box with 0.15 - $\AA$ point spacing centered at the nucleotide binding pocket. The Lamarckian Genetic Algorithm was used to search for lowenergy binding modes. Each simulation began with a randomly positioned ligand. Resulting orientations were evaluated visually in PyMOL with a preference for the cluster with the lowest predicted binding energy with multiple conformations.

Differential Scanning Fluorimetry. Thermal shift assays were performed using an Eppendorf Mastercycler RealPlex4 quantitative PCR instrument. Protein unfolding was monitored by fluorescence of SYPRO Orange dye with a 580-nm emission filter. Assay conditions were as follows: $75 \mathrm{mM}$ HEPES, $\mathrm{pH} 7.5,50 \mathrm{mM} \mathrm{KCl}, 5$ $\mathrm{mM} \mathrm{MgCl}_{2}, 2 \mathrm{mM}$ DTT, $5 \mu \mathrm{M}$ protein, and 25X SYPRO Orange dye. Samples were prepared in 96-well PCR plates at a volume of $25 \mu \mathrm{l}$. Samples were equilibrated to $15^{\circ} \mathrm{C}$ for 5 minutes and then heated to $75^{\circ} \mathrm{C}$ at a constant rate over 30 minutes. Fluorescence measurements were obtained every 8.15 seconds. Five technical replicates of each protein were run in parallel 3 times, each on separate days. The derivative of the fluorescence signal with respect to temperature was calculated by RealPlex software (Eppendorf). Both raw fluorescence 
and derivative data for sets of technical replicates for each mutant were normalized to the highest value among the replicates and averaged. The melting temperature of each protein corresponds to the temperature at which the derivative curve reaches its maximum value. Statistics were performed comparing each mutant to wild-type CKM using a one-way ANOVA followed by a Dunnett multiple comparison test using GraphPad Prism.

Hydrogen-Deuterium Exchange Mass Spectrometry. Wildtype CKM and W211R were exchanged into $75 \mathrm{mM}$ HEPES, $\mathrm{pH} 7.5$, $50 \mathrm{mM} \mathrm{KCl}, 5 \mathrm{mM} \mathrm{MgCl}$, and $2 \mathrm{mM}$ DTT and concentrated to 250 $\mu \mathrm{M}$. Labeling reactions were performed by addition of $2 \mu \mathrm{l}$ of the protein solution to $38 \mu \mathrm{l}$ of labeling buffer: $75 \mathrm{mM}$ HEPES, $\mathrm{pH} 7.5,50$ $\mathrm{mM} \mathrm{KCl}, 5 \mathrm{mM} \mathrm{MgCl}$, and $2 \mathrm{mM}$ DTT in $99 \%$ deuterium oxide [94\% deuterium oxide $(\mathrm{v} / \mathrm{v})$ labeling reaction]. Labeling was quenched by addition of $20 \mu \mathrm{l}$ ice-cold $10 \%$ formic acid, producing a solution with a $\mathrm{pH}$ of 2.5, and placed on ice to minimize back exchange. Most labeling reactions were incubated at $25^{\circ} \mathrm{C}$ and were quenched after 10 , $100,1000,10,000$, and 100,000 seconds. Another set of reactions were performed on ice for 10 seconds, which is approximately equivalent to 1 second of labeling time at $25^{\circ} \mathrm{C}$ based on the Arrhenius equation as previously described (Bai et al., 1993; Englander, 2006; Oganesyan et al., 2018). After quenching, proteins were proteolytically digested by addition of $20 \mu \mathrm{l}$ of $500 \mu \mathrm{M}$ porcine pepsin in $50 \mathrm{mM}$ $\mathrm{HCl}$ and incubated on ice for 5 minutes. Samples were then flash-frozen and stored at $-80^{\circ} \mathrm{C}$ until analysis. Labeling reactions were performed in triplicate. Unlabeled control samples were prepared similarly in protic solvent for each protein for the purposes of peptide identification and nondeuterated mass-spectrum analysis.

Samples were analyzed using a Dionex Ultimate 3000 uHPLC connected to a Q-Exactive quadrupole-orbitrap hybrid mass spectrometer (Thermo Fisher Scientific, Bremen, Germany). Samples were individually thawed on ice immediately prior to injection into the system. Mobile phase A was $0.1 \%$ formic acid in water, and mobile phase B was $0.1 \%$ formic acid in acetonitrile. Peptides were separated on a Waters Acquity peptide BEH C18 column $(2.1 \mathrm{~mm} \times 100$ $\mathrm{mm}, 1.7 \mu \mathrm{m}$ ) (Waters Corporation, Milford, MA) at a flow rate of 0.2 $\mathrm{ml} / \mathrm{min}$ using the following gradient: $0 \% \mathrm{~B}$ from 0 to 2 minutes, $0 \%-35 \%$ B from 2 to 4 minutes, $35 \%-90 \%$ B from 4 to 6 minutes, $90 \%$ B from 6 to 7.2 minutes, $90 \%-0 \%$ B from 7.2 to 7.5 minutes, $0 \%-5 \%$ B from 7.5 to 9 minutes, and $5 \%$ B from 9 to 10 minutes. To minimize back exchange, mobile phases were kept on ice, and the column compartment of the uHPLC system was kept at $5^{\circ} \mathrm{C}$. Peptides were ionized by heated electrospray ionization with a spray voltage of $3000 \mathrm{~V}$, capillary temperature of $175^{\circ} \mathrm{C}$, sheath gas flow rate of $35 \mathrm{Arb}$, and auxiliary gas flow rate of $8 \mathrm{Arb}$. The mass spectrometer was operated in positive ion mode. Peptide identification samples were analyzed using data-dependent MS/MS scans (dd-MS ${ }^{2}$ ) for the top-10 abundant ions at a resolution of 140,000 full width at half maximum with a dynamic exclusion of 6 seconds and applied a normalized collision energy of 27. MS/MS fragments were detected at a resolution of 35,000 full width at half maximum. Detection of deuterated peptides was performed using a full-MS scan identical to that portion of the dd-MS ${ }^{2}$ method.

Peptide identity, charge state, and retention time were determined using Proteome Discoverer v2.4 (Thermo Fisher Scientific, Bremen, Germany). The dd-MS ${ }^{2}$ data set was searched using Sequest HT to search manually modified human CKM protein FASTA sequence files. Peptides covering $100 \%$ of each protein sequence were able to be detected in nondeuterated controls. The presence of the W211R mutation did not significantly alter the digest peptides detected, so only peptides found in both control experiments (or for the peptides containing the mutant residue, the analogous peptides spanning from the same start and stop position) were used for downstream analyses. Deuterium incorporation calculations were performed using the HDX Workbench software platform (Pascal et al., 2012). Deuterium incorporation for each peptide was determined by the centroid of the isotopic distribution of each corresponding spectrum at each timepoint. These data were used to further calculate the average deuterium incorporation at each residue across all timepoints. The difference in these values between WT and W211R were visualized on a previously solved CKM crystal structure (PDB 1I0E) by coloring each position as a function of the magnitude of the difference in percent deuterium incorporation (Shen et al., 2001). The deuterium incorporation data for each peptide are summarized in Supplemental Document 2, in accordance with previous recommendations (Masson et al., 2019).

\section{Results}

CKM Mutants Differentially Reduce In Vitro Activity in a Substrate-Dependent Manner. To test whether naturally occurring mutations decrease the ability of CKM to phosphorylate TFV-MP, in vitro assays were performed using recombinantly expressed mutants. Purified proteins were incubated with phosphocreatine and TFV-MP, and TFV-DP production was measured by uHPLC-MS/MS. It was found that 10 of the 15 mutants resulted in statistically significant reductions in TFV-DP formation compared with WT (Fig. 1). The four mutations that produced the largest effect were R130H, R132C, W211R, and N286I; all reduced TFV-DP production to less than $5 \%$ of that of WT. Additionally, the mutations T35I, R43Q, I92M, H97Y, F169L, and Y173C reduced TFV-DP formation to $14 \%-25 \%$ of WT levels. Of the 10 mutations that reduced TFV-DP formation to less than $25 \%$ of that of WT, 8 were previously predicted by SIFT and PolyPhen to be deleterious to enzyme function (Lade et al., 2015; Figueroa et al., 2018a,b). Interestingly, R43Q and F169L mutations were predicted to be benign but resulted in decreased TFV-MP phosphorylation. Additionally, T52N, F250S, N274Y, and L317M were predicted to have decreased activity but did not exhibit significant reductions in TFV-DP production.

The impact of these mutations on the canonical CKM activity analogous to the TFV-MP phosphorylation reaction was determined using a similar assay in which the phosphorylation of the endogenous substrate ADP to form ATP was measured. Just four mutations-R130H, R132C, W211R, and N286I-led to significant reductions in ATP formation $(<30 \%$ of WT) (Fig. 2). Among these four mutations, $\mathrm{R} 130 \mathrm{H}$ and R132C had the lowest activity, reducing ATP formation to

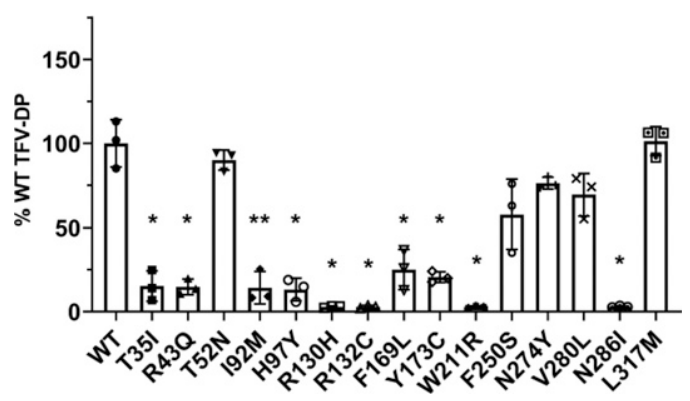

Fig. 1. Impact of naturally occurring CKM mutations on in vitro TFVMP phosphorylation. Recombinantly expressed and purified CKM mutants were incubated with TFV-MP and phosphocreatine, and TFVDP formation was measured by uHPLC-MS/MS. Chromatographic peak height was used as the measurement for relative TFV-DP formation. All data were normalized to the avg. WT value. The assay was performed with $n=3$, and error bars represent standard deviation. Statistics were performed comparing each mutant to wild-type CKM using a one-way ANOVA followed by a Dunnett multiple-comparisons test. Significance was set as follows: *, $P \leq 0.05$; **, $P \leq 0.01$; ***, $P \leq$ 0.001 . 


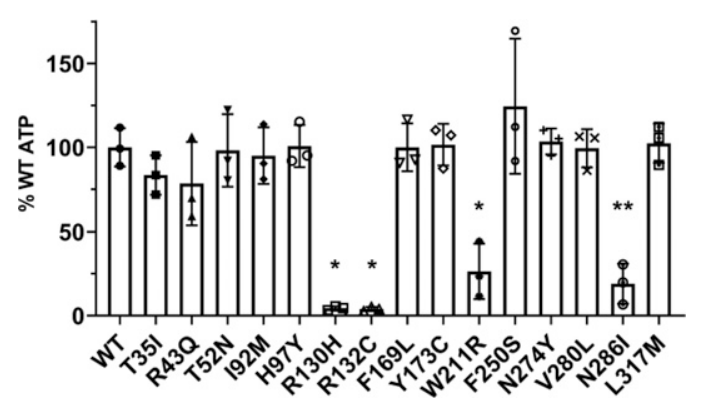

Fig. 2. Impact of naturally occurring CKM mutations on in vitro ADP phosphorylation. Recombinantly expressed and purified CKM mutants were incubated with ADP and phosphocreatine, and ATP formation was measured by uHPLC-MS/MS. Chromatographic peak height was used as the measurement for relative ATP formation. All data were normalized to the avg. WT value. The assay was performed with $n=3$, and error bars represent standard deviation. Statistics were performed comparing each mutant to wild-type CKM using a one-way ANOVA followed by a Dunnett multiple-comparisons test. Significance was set as follows: *, $P \leq 0.05$; ** $P \leq 0.01 ; * * *, P \leq 0.001$.

less than 5\% of WT levels. The other mutations tested did not result in significant changes in ATP formation. The four mutations that had the greatest reduction in this activity also produced the strongest effect on TFV-MP phosphorylation activity. Interestingly, although all mutants with reduced ATP formation were predicted to be deleterious to enzyme function, the majority of the mutations predicted to reduce CKM activity did not lead to reductions in ADP phosphorylation.

Further study of the reverse canonical reaction-dephosphorylation of ATP to ADP-was performed using an enzyme-coupled kinetic assay with varying concentrations of ATP. The same four mutations with reduced ADP phosphorylation activity-R130H, R132C, W211R, and N286Iresulted in the largest decreases in catalytic efficiency, all falling to less than $4 \%$ of WT $\mathrm{k}_{\mathrm{cat}} / \mathrm{K}_{\mathrm{M}}$ (Table 1). Additionally, the $\mathrm{H} 97 \mathrm{Y}$ and $\mathrm{Y} 173 \mathrm{C}$ mutations led to reductions in catalytic efficiency to $6 \%$ and $21 \%$ of WT, respectively. The L317M mutant displayed an increased catalytic efficiency (244\% of WT). $\mathrm{R} 132 \mathrm{C}$ resulted in the largest decrease in $\mathrm{k}_{\mathrm{cat}} / \mathrm{K}_{\mathrm{M}}(0.9 \%$ of WT). For this mutant, at the highest concentrations of ATP used, the initial rate remained linear as a function of substrate concentration, so the data were fit to a linear regression rather than the Michaelis-Menten equation, and thus separate $\mathrm{k}_{\mathrm{cat}}$ and $\mathrm{K}_{\mathrm{M}}$ fitted parameters were unable to be obtained for this mutant (Supplemental Fig. 1). For the other mutants with reduced activity in this assay, the reduction in catalytic efficiency was mostly $\mathrm{K}_{\mathrm{M}^{-}}$driven. The greatest reduction in $\mathrm{k}_{\text {cat }}$ was by $60 \%$, whereas three mutants resulted in a 13-fold or greater increase in $\mathrm{K}_{\mathrm{M}}$.

Molecular Docking Simulations ADP and TFV-MP Dock to CKM in Similar Conformations. To compare how CKM might interact with ADP and TFV-MP, docking simulations were performed. Although a crystal structure of rabbit CKM bound to a transition state analog complex set of ligands that includes ADP has been solved, no such cocrystal structure with human CKM has been described (Ohren et al., 2007). Rabbit and human CKM are similar in sequence (97\% identical sequences); thus, their structures are also likely similar. Although a human CKM structure has been solved in the absence of ligands, it is missing some residues near
TABLE 1

Michaelis-Menten kinetics parameters of naturally occurring CKM mutations Recombinantly expressed and purified CKM mutants were incubated with creatine and variable ATP conc. along with a pyruvate kinase/lactate dehydrogenase system. ATP dephosphorylation was measured by changes in NADH conc. monitored by absorbance at $340 \mathrm{~nm}$ that were the result of the coupled lactate dehydrogenase reaction. The assay was performed with $\mathrm{n}=3$, and the data were globally fitted to the Michaelis-Menten equation. Kinetic parameters are presented as fitted values or calculated values based on fitted parameters with standard error. A linear regression produced the best fit of the R132C data, thus only a fitted $\mathrm{k}_{\mathrm{cat}} / \mathrm{K}_{\mathrm{m}}$ value was able to be obtained and separate $\mathrm{k}_{\mathrm{cat}}$ and $\mathrm{K}_{\mathrm{m}}$ values are reported as N/A (not applicable).

\begin{tabular}{lccc}
\hline Mutant & $\mathrm{k}_{\text {cat }}\left(\mathrm{s}^{-1}\right)$ & $\mathrm{K}_{\mathrm{m}}(\mathrm{mM})$ & $\mathrm{k}_{\mathrm{cat}} / \mathrm{K}_{\mathrm{m}}\left(\mathrm{M}^{-1} \mathrm{~s}^{-1}\right)$ \\
\hline WT & $29.15 \pm 1.50$ & $1.271 \pm 0.289$ & $22,930 \pm 5340$ \\
T35I & $26.96 \pm 1.62$ & $1.870 \pm 0.460$ & $14,410 \pm 3650$ \\
R43Q & $21.93 \pm 0.99$ & $2.090 \pm 0.375$ & $10,490 \pm 1940$ \\
T52N & $22.39 \pm 1.24$ & $1.047 \pm 0.266$ & $21,390 \pm 5560$ \\
I92M & $20.19 \pm 1.39$ & $1.406 \pm 0.419$ & $14,290 \pm 4370$ \\
H97Y & $11.68 \pm 1.23$ & $8.513 \pm 2.577$ & $1371 \pm 439$ \\
R130H & $13.41 \pm 1.84$ & $16.49 \pm 5.34$ & $813.1 \pm 286.2$ \\
R132C & N/A & N/A & $210.5 \pm 12.4$ \\
F169L & $19.13 \pm 1.73$ & $1.220 \pm 0.492$ & $15,680 \pm 6480$ \\
Y173C & $14.37 \pm 0.79$ & $3.009 \pm 0.615$ & $4775 \pm 1011$ \\
W211R & $16.74 \pm 3.48$ & $30.35 \pm 12.28$ & $551.6 \pm 250.9$ \\
F250S & $24.53 \pm 1.63$ & $0.9823 \pm 0.3042$ & $24,960 \pm 7910$ \\
N274Y & $22.52 \pm 1.19$ & $1.237 \pm 0.291$ & $18,200 \pm 4380$ \\
V280L & $25.09 \pm 1.39$ & $1.423 \pm 0.340$ & $17,630 \pm 4330$ \\
N286I & $15.20 \pm 2.88$ & $22.33 \pm 9.09$ & $680.7 \pm 305.7$ \\
L317M & $40.98 \pm 2.85$ & $0.7334 \pm 0.2534$ & $55,880 \pm 19,700$ \\
\hline
\end{tabular}

the active site (Shen et al., 2001). Additionally, previous studies of human brain-type creatine kinase have described conformational changes upon ligand binding (Bong et al., 2008). Thus, the rabbit cocrystal structure served as a better template for the docking receptor for studying ligand binding. A homology model of human CKM based on the rabbit transition state analog complex structure was generated and used as the receptor for docking simulations.

The docking of ADP to CKM resulted in a low-energy conformation that closely reproduced the conformation of ADP solved in the cocrystal rabbit CKM structure (Fig. 3A). All functional groups were highly aligned, although other than the phosphates, the docked structure was somewhat shifted while maintaining a similar orientation. The docked ADP is also positioned to maintain the same protein-ligand interactions as the cocrystal structure ADP. This high degree of agreement validates that the homology model closely resembles the rabbit CKM structure and that the docking parameters used were appropriate.

Docking simulations with TFV-MP as the ligand predicted a similar binding conformation (Fig. 3B). The lowest-energy binding conformation of docked TFV-MP has both the adenosine rings and the phosphonate and phosphate in close alignment to both the rabbit cocrystal structure and docked ADP. Key contacts with active-site arginine residues and H296 were maintained, whereas the T322 contact was lost, as this residue interacts with a ribose hydroxyl group on ADP that is not present in TFV-MP.

Differential Scanning Fluorimetry CKM Mutations Lead to a Reduction in Thermal Stability. The effect of the naturally occurring mutations on the thermal stability of CKM was determined by a thermal shift assay. It was found that T35I, R43Q, I92M, and W211R mutants had the largest reduction in melting temperatures compared with WT (Fig. 
A

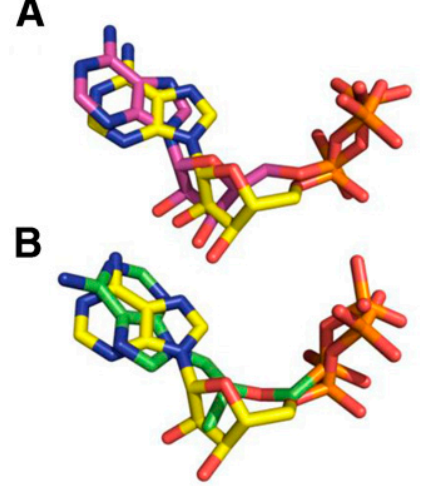

C

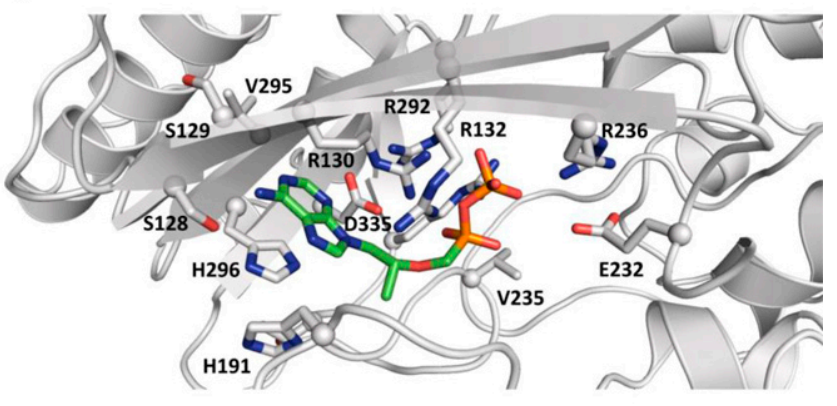

Fig. 3. Molecular docking of ADP and TFV-MP to CKM. (A) The lowest-energy docking conformation of ADP (purple) aligns well with the ADP from a solved cocrystal structure (yellow). (B) The lowest-energy docking conformation of TFV-MP (green) also closely aligns to the solved ADP positioning, suggesting TFV-MP binds to CKM in a similar position and orientation as the endogenous substrate. (C) Representation of CKM active-site residues likely involved in TFV-MP binding based on the docked TFV-MP pose.

4; Supplemental Fig. 2). The $\mathrm{T}_{\mathrm{m}}$ values of the T35I and I92M mutants were about $8^{\circ} \mathrm{C}$ lower than WT, whereas $\mathrm{R} 43 \mathrm{Q}$ and $\mathrm{W} 211 \mathrm{R}$ led to reductions by $13.5^{\circ} \mathrm{C}$ and $10^{\circ} \mathrm{C}$, respectively. The T52N and Y173C mutants both had smaller reductions in $\mathrm{T}_{\mathrm{m}}$, with both decreasing by $3.6^{\circ} \mathrm{C}$. All other mutants had $\mathrm{T}_{\mathrm{m}}$ values within $2^{\circ} \mathrm{C}$ of WT, with both small increases and decreases in $\mathrm{T}_{\mathrm{m}}$ being observed.

The four mutations that resulted in the largest decrease in melting temperature also displayed reduced TFV-MP phosphorylation activity. Interestingly, T35I, R43Q, and I92M did not lead to large reductions of canonical CKM activities, whereas W211R showed significantly reduced activity in all assays. Additionally, in contrast to W211R, the other three mutants with low activity toward each substrate (R130H, R132C, and $\mathrm{N} 286 \mathrm{I}$ ) resulted in relatively small changes in $\mathrm{T}_{\mathrm{m}}$, suggesting the mutations exert their effects by different mechanisms.

The W211R Mutation Results in Both Local and Distant Changes in Deuterium Incorporation. The impact of the W211R mutation on CKM structure was determined by hydrogen-deuterium exchange mass spectrometry (HDX-MS) comparing the deuterium uptake for this mutant to WT. Deuterium uptake data were obtained for both proteins on

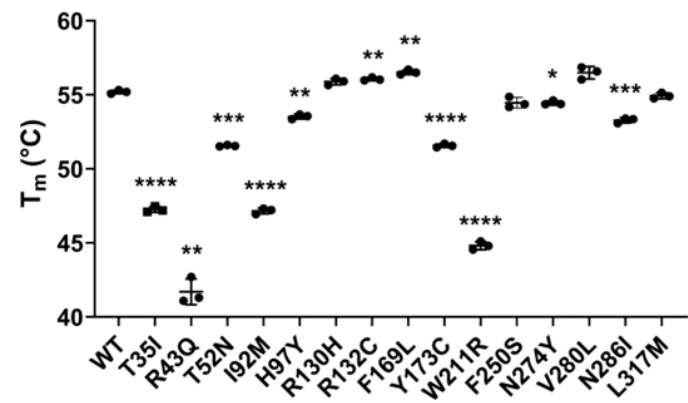

Fig. 4. Thermal stability comparison of naturally occurring CKM mutants. Recombinantly expressed and purified CKM mutants were incubated in activity assay buffer and fluorescent SYPRO Orange dye in a quantitative PCR instrument, and temperature was increased from $15^{\circ} \mathrm{C}$ to $75^{\circ} \mathrm{C}$ over 30 minutes. The temperature at which the maximum of the derivative of fluorescence intensity with respect to temperature was calculated to be the $\mathrm{T}_{\mathrm{m}}$. The assay was performed with $n=3$ sets of five averaged technical replicates, and error bars represent standard deviation. Statistics were performed comparing each mutant to wild-type CKM using a one-way ANOVA followed by a Dunnett multiple-comparisons test. Significance was set as follows: *, $P \leq 0.05 ; * *, P \leq 0.01 ; * * *, P \leq 0.001$, ****, $P \leq 0.0001$ peptides that cover $93 \%$ of the protein sequence (Supplemental Document 2), with the majority of the missing sequence coverage coming from a gap between positions 39 and 56. All other gaps in sequence coverage were fewer than 5 consecutive amino acids. The average deuterium incorporation for each peptide across the full set of labeling reaction times was calculated, and the data from overlapping peptides were combined to calculate more specific average incorporation values for each residue. To compare deuterium incorporation between WT and W211R, the average percent deuterated value at each position for the wild-type protein data was subtracted from the corresponding $\mathrm{W} 211 \mathrm{R}$ value, such that $\Delta \% \mathrm{D}=\% \mathrm{D}_{\mathrm{W} 211 \mathrm{R}}-$ $\% \mathrm{D}_{\mathrm{WT}}$, with each $\% \mathrm{D}$ parameter being the average percent deuteration.

It was found that multiple regions of the protein had moderate changes in deuterium incorporation, with $\Delta \% \mathrm{D}$ values ranging from -9 to 7 . These changes were found to occur in multiple parts of the three-dimensional structure of CKM (Fig. 5). A decrease in deuterium incorporation with a $\Delta \% \mathrm{D}$ of -5 was observed in the region of the sequence containing position 211 and spanning from positions 205 through 225 . A larger decrease in uptake was observed at positions 24-32 $(\Delta \% \mathrm{D}$ of -9$)$. An increase in deuterium incorporation with a $\Delta \% \mathrm{D}$ of 7 was observed at positions $36-38$, and a $\Delta \% \mathrm{D}$ of 4 was observed at positions 132-159. Additional regions with $\Delta \% \mathrm{D}$ values between 3.5 and 5 were observed at positions 228-237 and 302-312. Although the W211R mutation resulted in changes in local deuterium incorporation (positions 205-225 and 228-237), it also led to changes in uptake to a similar or greater degree at more distant regions of the structure, including toward the N terminus (positions 24-32 and 26-38) and into the active site (positions 132-159).

\section{Discussion}

These results demonstrate that naturally occurring CKM mutations impact both canonical biologic and pharmacological activities in vitro in a substrate-dependent manner. Of the 15 mutations studied, 10 resulted in a reduction in TFVMP phosphorylation activity, whereas only 4 caused a decrease in ADP phosphorylation, and 6 decreased ATP dephosphorylation activity. The same four mutations that decreased ADP phosphorylation led to the greatest relative 


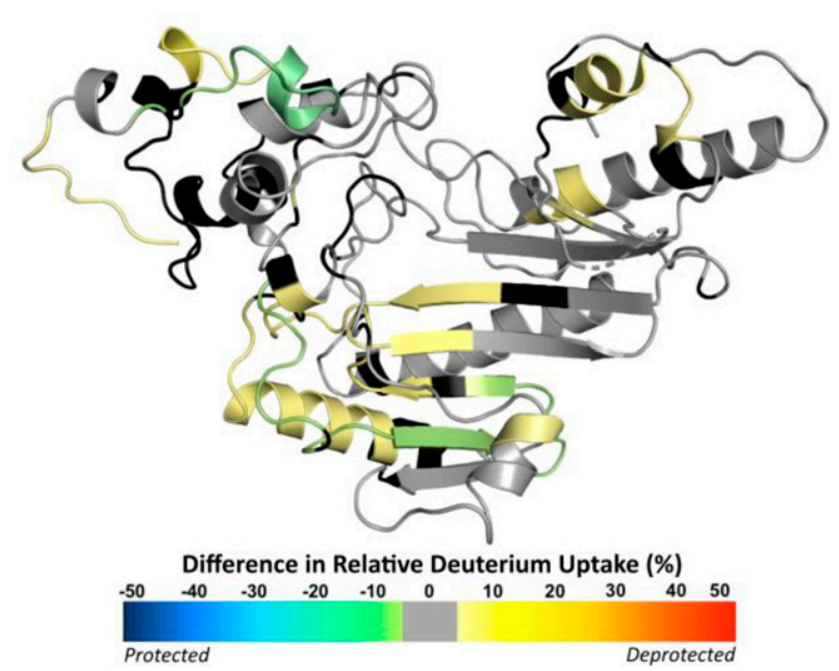

Fig. 5. Positions in the CKM structure in which the W211R mutation results in a change in deuterium incorporation compared with WT. HDX-MS $\Delta \% \mathrm{D}$ values were mapped to PDB $1 \mathrm{IOE}$ according to the displayed color scale. Residues wherein no deuterium incorporation data were obtained for either protein sample were colored black. Positions with small changes in deuterium incorporation $(\Delta \% \mathrm{D}-2.5 \%$ to $2.5 \%)$ were colored gray. There was a decrease in deuterium incorporation between positions 205 and 226 and an increase in deuterium incorporation between positions 228 and 237. More distant from the site of the mutation, differences were also observed toward the $\mathrm{N}$ terminus (positions 20-38) and at active site (positions 132-159).

reduction in activity in all three assays. Although all mutations that reduced canonical CKM function also displayed reduced pharmacological function, the inverse is not true. Four mutations reduced TFV-DP formation to less than $30 \%$ of that of WT while not significantly decreasing ADP phosphorylation or ATP dephosphorylation kinetics. This suggests people carrying these mutations may have no pathophysiological phenotype as a result of the CKM mutation yet may have reduced ability to form TFV-DP in colon tissue as readily as others.

The colon is a putative site of HIV infection, and it has been demonstrated that intracellular colorectal TFV-DP levels reduce infectibility in a concentration-dependent manner in an ex vivo biopsy model (Richardson-Harman et al., 2014). Thus, low-activity mutations could potentially lead to failure of TFVbased PrEP and could especially pose a problem for the ondemand dosing PrEP strategies being developed, in which the rate of TFV-DP formation is of greater importance than in typical PrEP strategies (Molina et al., 2015; Antoni et al., 2020). Serum creatine kinase activity measurements are routine clinical assessments performed especially in instances of muscle damage (Clarkson et al., 2006; Magal et al., 2010). However, the observed substrate-specific effect of mutations suggests a lack of predictive power of these assays for TFV activation.

Targeted genotyping of CKM from a pharmacogenomic perspective is still in its nascent stage. Of the 15 mutations studied, 8 do not have reported minor allele frequency values in the dbSNP database (Sherry et al., 1999). The seven mutations with reported frequencies-T35I, R43Q, R130H, R132C, F169L, V280L, and N286I-range from 0.000005 to 0.00016 . Although the frequencies of individual mutations are rather low, the presence of multiple mutations with reduced activity suggests the prevalence of reduced TFV-MP phosphorylation activity may be greater than the frequency of any single mutation. Studies have yet to be performed to investigate the relationship between CKM variants and drug disposition in vivo. However, two CKM mutations, D54G and E83G, have been associated with reduced serum creatine kinase activity, demonstrating the potential for CKM mutations to impact physiology (Yamamichi et al., 2001; Dubé et al., 2014).

Given the substrate-dependent effect observed in the in vitro activity assays, the results of the molecular docking simulations are surprising. Docking simulations predict both ADP and TFV-MP likely bind the CKM nucleotide binding site in the same space and orientation. These conformations, especially that of ADP, also align well with the positioning of ADP in a previously described CKM cocrystal structure (Ohren et al., 2007). Although the adenosine rings of TFVMP are flipped compared with ADP, the ring system occupies the same plane and can $\pi-\pi$ stack with H296. Additionally, the N1 and N6 adenosine amines switched positions and maintain the contact with S128. The $\alpha$ and $\beta$ phosphates of $\mathrm{ADP}$ align well with the phosphonate and phosphate of TFVMP and maintain the same predicted interactions with a cluster of active-site arginine residues. Indeed, the $\mathrm{R} 130 \mathrm{H}$ and $\mathrm{R} 132 \mathrm{C}$ mutations in this region displayed decreased activity toward all substrates studied. The section of the molecules in between the phosphates and the adenosine rings does differ in structure and in predicted contacts with residues of CKM, so it is possible that these differences have some role in conferring the substrate-specific effects.

However, the majority of mutations that decreased TFVMP phosphorylation are not in the active site of CKM. It is likely that these mutations are impacting function through other indirect mechanisms. It was previously found that CKM has a weaker binding affinity for TFV-MP than ADP (Varga et al., 2013). Thus, the binding may be less tolerant of small changes in global protein structure that result from these mutations. The thermal shift assay results indicate that most of the mutations resulted in statistically significant but small changes in melting temperature. None of the mutations resulted in a large increase in the melting temperature. The large reduction of melting temperature observed for the T35I, R43Q, I92M, and W211R mutants indicates a decrease in thermal stability. All four of these mutations displayed decreased TFV-MP phosphorylation activity, whereas just W211R decreased the ADP- and ATP-related activities. The majority of the mutations that reduced TFV-MP phosphorylation but not the canonical activities did not result in a large decrease in melting temperature. Thus, whereas decreased thermal stability may be related to structural changes resulting from some of the mutations that impact CKM function, it does not explain the mechanism by which several other mutations decrease the pharmacological function. Further biochemical and structural experiments will be needed if the exact mechanism by which each mutation impacts activity is to be determined.

Regarding the four mutations that displayed low activity in all three activity assays, three (R130H, R132C, and N286I) are at positions in the CKM active site, whereas $\mathrm{W} 211 \mathrm{R}$ is outside of the active site. R130 and R132 are implicated by biochemical assays and crystal structures to be involved in nucleotide binding and phosphoryl transfer (Wood et al., 1998; Ohren et al., 2007). Indeed, both are predicted to be involved in both ADP and TFV-MP binding by the molecular docking simulations (Fig. 3C). N286 is 
implicated by crystal structures to be involved in the binding of creatine/phosphocreatine. All three of these active-site positions are highly conserved in CKM across species and also among other creatine kinase isoenzymes (Mühlebach et al., 1994). It is likely these mutations result in loss of key interactions to substrate binding that impacts the activity of the enzyme toward all three substrates studied. Additionally, none of these three mutations resulted in a change in melting temperature by more than $2^{\circ} \mathrm{C}$ in either direction, indicating that changes to thermal stability are not likely the mechanism by which these mutations result in decreased activity.

Interestingly, position 211 is not located in the active site but results in reduced activity in all three in vitro assays to a similar magnitude as the active site mutations. This mutation did lead to a $10^{\circ} \mathrm{C}$ reduction in melting temperature, indicating that this mutation impacts the thermal stability of CKM, which may relate to the mechanism by which its activity is reduced. Because of the large magnitude of its effect on enzyme activity, further elucidation of the structural impact of this mutation was determined by HDX-MS. The results show this mutation had a mild impact on the deuterium incorporation compared with WT in multiple regions of the three-dimensional CKM structure. Although these data cannot conclusively demonstrate a change in peptide backbone structure, they can indicate regions in which the peptide backbone became more or less accessible to deuterium exchange in solution, which relates to changes in hydrogen bonding networks, such as secondary structure and overall flexibility of that region. A decrease in deuterium incorporation was observed at positions near position 211 by sequence and three-dimensional folding. Additionally, changes in deuterium incorporation were also observed at regions more distant from the part of the structure containing position 211 . This includes segments closer to the $\mathrm{N}$ terminus (positions 24-38) and, notably, positions 132-159, which include residues in and around the active-site pocket. That the W211R mutation impacts deuterium exchange in multiple parts of the structure indicates a change in structure or in-solution dynamics that may relate to the mechanism by which this mutation leads to decreased function. Looking previously solved CKM crystal structures, W211 is a bulky, hydrophobic residue that points inward into the hydrophobic core of the structure. A mutation to arginine is likely to prevent the side chains in this region and possibly the peptide backbone from residing in the usual position, potentially forcing a change in local structure if not a more global effect.

In conclusion, it was demonstrated that naturally occurring mutations to muscle-type creatine kinase are capable of reducing the ability of the enzyme to phosphorylate TFV-MP to TFV-DP, a function that is important to the efficacy of PrEP. Interestingly, most of these mutations were tolerated in assays with the endogenous substrates of CKM. This substrate-specific effect suggests that people may possess CKM mutations that do not impact normal physiology but may lead to unfavorable outcomes if one was prescribed TFV-based PrEP. Additionally, typical clinical measures of creatine kinase activity would not likely be fully predictive of potential for PrEP failure. The results of this study indicate that it is possible for someone to have a mutation that does not impact the canonical function measured in this assay while potentially conferring reduced TFV-MP phosphorylation activity.
This perspective is important to future pharmacogenomic studies, especially in instances of nontraditional drug-metabolizing enzymes that have other physiologic functions.

\section{Authorship Contributions}

Participated in research design: Mosher, Eberhard, Bumpus. Conducted experiments: Mosher.

Performed data analysis: Mosher, Eberhard, Bumpus.

Wrote or contributed to the writing of the manuscript: Mosher, Eberhard, Bumpus.

\section{References}

Adzhubei IA, Schmidt S, Peshkin L, Ramensky VE, Gerasimova A, Bork P, Kondrashov AS, and Sunyaev SR (2010) A method and server for predicting damaging missense mutations. Nat Methods 7:248-249.

Anderson PL, Glidden DV, Liu A, Buchbinder S, Lama JR, Guanira JV, McMahan V, Bushman LR, Casapía M, Montoya-Herrera O, et al.; iPrEx Study Team (2012) Emtricitabine-tenofovir concentrations and pre-exposure prophylaxis efficacy in men who have sex with men. Sci Transl Med 4:151ra125.

Anton PA, Cranston RD, Kashuba A, Hendrix CW, Bumpus NN, Richardson-Harman N, Elliott J, Janocko L, Khanukhova E, Dennis R, et al. (2012) RMP-02/MTN-006: a phase 1 rectal safety, acceptability, pharmacokinetic, and pharmacodynamic study of tenofovir $1 \%$ gel compared with oral tenofovir disoproxil fumarate. AIDS Res Hum Retroviruses 28:1412-1421.

Antoni G, Tremblay C, Delaugerre C, Charreau I, Cua E, Rojas Castro D, Raffi F, Chas J, Huleux T, Spire B, et al.; ANRS IPERGAY study group (2020) On-demand pre-exposure prophylaxis with tenofovir disoproxil fumarate plus emtricitabine among men who have sex with men with less frequent sexual intercourse: a posthoc analysis of the ANRS IPERGAY trial. Lancet HIV 7:e113-e120.

Baeten JM, Donnell D, Ndase P, Mugo NR, Campbell JD, Wangisi J, Tappero JW, Bukusi EA, Cohen CR, Katabira E, et al.; Partners PrEP Study Team (2012) Antiretroviral prophylaxis for HIV prevention in heterosexual men and women. $N$ Engl $J$ Med 367:399-410

Bai Y, Milne JS, Mayne L, and Englander SW (1993) Primary structure effects on peptide group hydrogen exchange. Proteins 17:75-86.

Bong SM, Moon JH, Nam KH, Lee KS, Chi YM, and Hwang KY (2008) Structural studies of human brain-type creatine kinase complexed with the ADP-Mg2+-NO3-creatine transition-state analogue complex. FEBS Lett 582:3959-3965.

Choopanya K, Martin M, Suntharasamai P, Sangkum U, Mock PA, Leethochawalit M, Chiamwongpaet S, Kitisin P, Natrujirote P, Kittimunkong S, et al.; Bangkok Tenofovir Study Group (2013) Antiretroviral prophylaxis for HIV infection in injecting drug users in Bangkok, Thailand (the Bangkok Tenofovir Study): a randomised, double-blind, placebo-controlled phase 3 trial. Lancet 381:2083-2090.

Chou R, Evans C, Hoverman A, Sun C, Dana T, Bougatsos C, Grusing S, and Korthuis PT (2019) Preexposure prophylaxis for the prevention of HIV infection: evidence report and systematic review for the US preventive services task force. JAMA 321:2214-2230.

Clarkson PM, Kearns AK, Rouzier P, Rubin R, and Thompson PD (2006) Serum creatine kinase levels and renal function measures in exertional muscle damage. Med Sci Sports Exerc 38:623-627.

Dubé MP, Zetler R, Barhdadi A, Brown AM, Mongrain I, Normand V, Laplante N, Asselin G, Zada YF, Provost S, et al. (2014) CKM and LILRB5 are associated with serum levels of creatine kinase. Circ Cardiovasc Genet 7:880-886.

Englander SW (2006) Hydrogen exchange and mass spectrometry: a historical perspective. J Am Soc Mass Spectrom 17:1481-1489.

Figueroa DB, Madeen EP, Tillotson J, Richardson P, Cottle L, McCauley M, Landovitz RJ, Andrade A, Hendrix CW, Mayer KH, et al. (2018a) Genetic variation of the kinases that phosphorylate tenofovir and emtricitabine in peripheral blood mononuclear cells. AIDS Res Hum Retroviruses 34:421-429.

Figueroa DB, Tillotson J, Li M, Piwowar-Manning E, Hendrix CW, Holtz TH, Bokoch K, Bekker LG, van Griensven F, Mannheimer S, et al. (2018b) Discovery of genetic variants of the kinases that activate tenofovir among individuals in the United States, Thailand, and South Africa: HPTN067. PLoS One 13:e195764.

Gasteiger E, Gattiker A, Hoogland C, Ivanyi I, Appel RD, and Bairoch A (2003) ExPASy: The proteomics server for in-depth protein knowledge and analysis. Nucleic Acids Res 31:3784-3788.

GBD 2017 HIV collaborators (2019) Global, regional, and national incidence, prevalence, and mortality of HIV, 1980-2017, and forecasts to 2030, for 195 countries and territories: a systematic analysis for the Global Burden of Diseases, Injuries, and Risk Factors Study 2017. Lancet HIV 6(12): e831-e859.

Grant RM, Lama JR, Anderson PL, McMahan V, Liu AY, Vargas L, Goicochea P, Casapía M, Guanira-Carranza JV, Ramirez-Cardich ME, et al.; iPrEx Study Team (2010) Preexposure chemoprophylaxis for HIV prevention in men who have sex with men. N Engl J Med 363:2587-2599.

Gunawardana M, Remedios-Chan M, Miller CS, Fanter R, Yang F, Marzinke MA, Hendrix CW, Beliveau M, Moss JA Smith TJ, et al (2015) Pharmacokinetics of long-acting tenofovir alafenamide (GS-7340) subdermal implant for HIV prophylaxis. Antimicrob Agents Chemother 59:3913-3919.

Johnson KA (2019) New standards for collecting and fitting steady state kinetic data. Beilstein J Org Chem 15:16-29.

Joint United Nations Programme on HIV/AIDS (2020) UNAIDS Data 2020.

Klock HE, Koesema EJ, Knuth MW, and Lesley SA (2008) Combining the polymerase incomplete primer extension method for cloning and mutagenesis with microscreening to accelerate structural genomics efforts. Proteins 71:982-994.

Koch K, Chen Y, Feng JY, Borroto-Esoda K, Deville-Bonne D, Gallois-Montbrun S, Janin J, and Moréra S (2009) Nucleoside diphosphate kinase and the activation of 
antiviral phosphonate analogs of nucleotides: binding mode and phosphorylation of tenofovir derivatives. Nucleosides Nucleotides Nucleic Acids 28:776-792.

Krieger E, Joo K, Lee J, Lee J, Raman S, Thompson J, Tyka M, Baker D and Karplus K (2009) Improving physical realism, stereochemistry, and side-chain accuracy in homology modeling: Four approaches that performed well in CASP8. Proteins 77 Suppl 9(Suppl 9): 114-122.

Lade JM, To EE, Hendrix CW, and Bumpus NN (2015) Discovery of genetic variants of the kinases that activate tenofovir in a compartment-specific manner. EBioMedicine 2:1145-1152.

Louissaint NA, Cao YJ, Skipper PL, Liberman RG, Tannenbaum SR, Nimmagadda S, Anderson JR, Everts S, Bakshi R, Fuchs EJ, et al. (2013) Single dose pharmacokinetics of oral tenofovir in plasma, peripheral blood mononuclear cells, colonic tissue, and vaginal tissue. AIDS Res Hum Retroviruses 29:1443-1450.

Magal M, Dumke CL, Urbiztondo ZG, Cavill MJ, Triplett NT, Quindry JC, McBride JM, and Epstein Y (2010) Relationship between serum creatine kinase activity following exercise-induced muscle damage and muscle fibre composition. $J$ Sports $S c i$ 28:257-266.

Mandal S, Kang G, Prathipati PK, Zhou Y, Fan W, Li Q, and Destache CJ (2019) Nanoencapsulation introduces long-acting phenomenon to tenofovir alafenamide and emtricitabine drug combination: a comparative pre-exposure prophylaxis efficacy study against HIV-1 vaginal transmission. J Control Release 294:216-225.

Marrazzo JM, Ramjee G, Richardson BA, Gomez K, Mgodi N, Nair G, Palanee T, Nakabiito C, van der Straten A, Noguchi L, et al.; VOICE Study Team (2015) Tenofovirbased preexposure prophylaxis for HIV infection among African women. $N$ Engl J Med 372:509-518.

Masson GR, Burke JE, Ahn NG, Anand GS, Borchers C, Brier S, Bou-Assaf GM, Engen JR, Englander SW, Faber J, et al. (2019) Recommendations for performing, interpreting and reporting hydrogen deuterium exchange mass spectrometry (HDX-MS) experiments. Nat Methods 16:595-602.

McCormack S, Dunn DT, Desai M, Dolling DI, Gafos M, Gilson R, Sullivan AK Clarke A, Reeves I, Schembri G, et al. (2016) Pre-exposure prophylaxis to prevent the acquisition of HIV-1 infection (PROUD): effectiveness results from the pilot phase of a pragmatic open-label randomised trial. Lancet 387:53-60.

Molina JM, Capitant C, Spire B, Pialoux G, Cotte L, Charreau I, Tremblay C, Le Gall JM, Cua E, Pasquet A, et al.; ANRS IPERGAY Study Group (2015) On-demand preexposure prophylaxis in men at high risk for HIV-1 infection. N Engl J Med 373:2237-2246.

Morris GM, Huey R, Lindstrom W, Sanner MF, Belew RK, Goodsell DS, and Olson AJ (2009) AutoDock4 and AutoDockTools4: automated docking with selective receptor flexibility. J Comput Chem 30:2785-2791.

Mühlebach SM, Gross M, Wirz T, Wallimann T, Perriard JC, and Wyss M (1994) Sequence homology and structure predictions of the creatine kinase isoenzymes. Mol Cell Biochem 133-134:245-262.

Ng PC and Henikoff S (2001) Predicting deleterious amino acid substitutions. Genome Res 11:863-874.

O’Boyle NM, Banck M, James CA, Morley C, Vandermeersch T, and Hutchison GR (2011) Open Babel: an open chemical toolbox. J Cheminform 3:33.

Oganesyan I, Lento C, and Wilson DJ (2018) Contemporary hydrogen deuterium exchange mass spectrometry. Methods 144:27-42.

Ohren JF, Kundracik ML, Borders Jr CL, Edmiston P, and Viola RE (2007) Struc- tural asymmetry and intersubunit communication in muscle creatine kinase. Acta Crystallogr D Biol Crystallogr 63:381-389.

Pascal BD, Willis S, Lauer JL, Landgraf RR, West GM, Marciano D, Novick S, Goswami D, Chalmers MJ, and Griffin PR (2012) HDX workbench: software for the analysis of H/D exchange MS data. J Am Soc Mass Spectrom 23:1512-1521.

Richardson-Harman N, Hendrix CW, Bumpus NN, Mauck C, Cranston RD, Yang K, Elliott J, Tanner K, McGowan I, Kashuba A, et al. (2014) Correlation between compartmental tenofovir concentrations and an ex vivo rectal biopsy model of tissue infectibility in the RMP-02/MTN-006 phase 1 study. PLoS One 9:e111507.

Shen YQ, Tang L, Zhou HM, and Lin ZJ (2001) Structure of human muscle creatine kinase. Acta Crystallogr D Biol Crystallogr 57:1196-1200.

Sherry ST, Ward M, and Sirotkin K (1999) dbSNP-database for single nucleotide polymorphisms and other classes of minor genetic variation. Genome Res 9:677-679.

Thigpen MC, Kebaabetswe PM, Paxton LA, Smith DK, Rose CE, Segolodi TM, Henderson FL, Pathak SR, Soud FA, Chillag KL, et al - TDF2 Study Group (2012) Antiretroviral preexposure prophylaxis for heterosexual HIV transmission in Botswana. N Engl J Med 367:423-434.

Van Damme L, Corneli A, Ahmed K, Agot K, Lombaard J, Kapiga S, Malahleha M, Owino F, Manongi R, Onyango J, et al.; FEM-PrEP Study Group (2012) Preexposure prophylaxis for HIV infection among African women. $N$ Engl $J$ Med 367:411-422

Varga A, Gráczer E, Chaloin L, Liliom K, Závodszky P, Lionne C, and Vas M (2013) Selectivity of kinases on the activation of tenofovir, an anti-HIV agent. Eur $J$ Pharm Sci 48:307-315.

Wallimann T, Tokarska-Schlattner M, and Schlattner U (2011) The creatine kinase system and pleiotropic effects of creatine. Amino Acids 40:1271-1296.

Wallimann T, Wyss M, Brdiczka D, Nicolay K and Eppenberger HM (1992) Intracellular compartmentation, structure and function of creatine kinase isoenzymes in tissues with high and fluctuating energy demands: the 'phosphocreatine circuit' for cellular energy homeostasis. Biochem $J 281$ (Pt 1)(Pt 1): 21-40.

Waterhouse A, Bertoni M, Bienert S, Studer G, Tauriello G, Gumienny R, Heer FT, de Beer TAP, Rempfer C, Bordoli L, et al. (2018) SWISS-MODEL: homology modelling of protein structures and complexes. Nucleic Acids Res 46 (W1):W296-W303.

Wood TD, Guan Z, Borders Jr CL, Chen LH, Kenyon GL, and McLafferty FW (1998) Creatine kinase: essential arginine residues at the nucleotide binding site identified by chemical modification and high-resolution tandem mass spectrometry. Proc Natl Acad Sci USA 95:3362-3365.

Yamamichi H, Kasakura S, Yamamori S, Iwasaki R, Jikimoto T, Kanagawa S, Ohkawa J, Kumagai S, and Koshiba M (2001) Creatine kinase gene mutation in a patient with muscle creatine kinase deficiency. Clin Chem 47:1967-1973.

Address correspondence to: Namandjé N. Bumpus, Department of Pharmacology and Molecular Sciences, Johns Hopkins University School of Medicine, 725 North Wolfe St., Physiology 312, Baltimore, MD 21205. E-mail: nbumpus1@jhmi.edu 